\title{
Selective Filling and Sintering of Copper Nanoclusters for Interconnect
}

\author{
Kheng Chok Tee, Andreas Lassesson, Joris van Lith, Simon A. Brown, Jim G. Partridge, Monica Schulze, and \\ Richard J. Blaikie, Member, IEEE
}

\begin{abstract}
In copper interconnect technology, dielectric trenches are patterned, filled with copper, and polished. We report a cluster-based deposition technology that provides efficient trench filling and excellent selectivity between trenches and plateaus on damascene structures. The selectivity arises due to the propensity for reflection of clusters from the planar surfaces between trenches. Trenches of sub-200 $\mathrm{nm}$ widths, with various diffusion barriers and seed layers, and up to $5: 1$ aspect ratios have been completely filled with copper clusters. We also show that copper clusters can be sintered into a seed layer using hydrogen annealing. Thus, dense copper films within trenches are obtained. Preliminary results from planar samples show that the resistivity is around $2.3 \times 10^{-8} \Omega \mathrm{m}$
\end{abstract}

Index Terms-Cluster deposition, copper, damascene trench filling, integrated circuit interconnections, integrated circuit metallization, materials science and technology.

\section{INTRODUCTION}

$\mathbf{I}$ $\mathrm{N}$ advanced integrated circuit technology, transistors, and metal lines for the connections between them (known as interconnects) must now be fabricated with dimensions in the nanoscale regime [1]. In order to continue the progression to smaller dimensions and higher performance, many different challenges relating to interconnect processing must be solved [2]. For example, as the pitch decreases and the aspect ratio (depth to width) increases, it is difficult to fill damascene trenches with copper without voids. Key-hole formation within the damascene structures compromises the reliability of the interconnects [3]. Furthermore, the chemical mechanical polishing (CMP) processes used to remove excess copper after electroplating have encountered great challenges especially with the integration of ultralow-k dielectrics [2]. Minimizing or even eliminating copper CMP would be highly desirable from a process integration point of view. Selective copper deposition

Manuscript received December 19, 2006; revised April 5, 2007. This work was supported in part by Foundation for Research, Science and Technology (New Zealand) under a Technology Industry Fellowship. The review of this paper was arranged by Associate Editor G. Ramanath.

K. C. Tee, J. G. Partridge, and R. J. Blaikie are with Nanostructure Engineering Science and Technology (NEST) Group and MacDiarmid Institute for Advanced Materials and Nanotechnology, University of Canterbury, Christchurch 8140, New Zealand.

A. Lassesson, J. van Lith, and M. Schulze are with Nano Cluster Devices Ltd, Rutherford Building, University of Canterbury, Christchurch 8140, New Zealand.

S. A. Brown is with Nanostructure Engineering Science and Technology (NEST) Group and MacDiarmid Institute for Advanced Materials and Nanotechnology, University of Canterbury, Christchurch 8140, New Zealand. He is also with Nano Cluster Devices Ltd, Rutherford Building, University of Canterbury, Christchurch 8140, New Zealand (e-mail: simon.brown@canterbury.ac.nz).

Digital Object Identifier 10.1109/TNANO.2007.902643 into deep and narrow trenches and vias would therefore be an advantage for technological advancement; we present a cluster-based deposition technique that has this capability.

Metallic clusters, which are aggregates of metal atoms, have many interesting and unusual properties [4] but there has not been extensive investigation of their application in integrated circuit applications. In one recent case, it has been shown that, depending on the kinetic energy and the momentum of the nanoscale clusters, they can bounce or slide in a template yielding nanowires [5]. The transition from adhesion to reflection has been verified by molecular dynamics simulations [6]. Previously, the use of cluster beams for filling micrometer-scale holes with copper [7] and the metallization of nonplanar surfaces [8] have been demonstrated. However, to our knowledge, there is no previous demonstration of the filling with clusters of the high aspect ratio nanoscale trenches which are currently employed for interconnects by industry. In addition, we demonstrate selective deposition and the sintering of the deposited clusters into the seed layer to achieve dense metal lines. Sintering is achieved by simple molecular hydrogen annealing without the need to apply other advanced annealing techniques [9], [10]. The high surface/volume ratio of the clusters is expected to enhance surface melting and coalescence [11], [12] during this annealing process.

\section{EXPERIMENTAL PROCEDURE}

In this work, nanoscale copper clusters are generated in an ultrahigh-vacuum compatible system (operating pressure $\sim 10^{-6}$ torr) by inert gas aggregation (IGA) in a magnetron sputtering source using a $99.99 \%$ pure $\mathrm{Cu}$ target. Fig. 1(a) shows the schematic of the sputtering source and full details of the cluster deposition system have been presented elsewhere [13]. Argon gas $(<1 \mathrm{ppm}$ impurities) is introduced into the source chamber to generate a plasma, to assist in the cluster aggregation, and to transport the clusters towards the exit of the source. The clusters are accelerated by the gas as it expands through the source exit nozzle into the ultrahigh-vacuum chamber.

Approximately one-third of the clusters deposited onto the samples are neutral and roughly the same proportions are positively or negatively charged (as determined by the effect of an applied electric field on the measured deposition rate). Similar ratios were also measured in [14]. By applying electrical pulses to a pair of parallel deflector plates and measuring the delay signal in a Faraday cup, the velocity of the clusters was found to be $230 \pm 30 \mathrm{~m} / \mathrm{s}$ at $800 \mathrm{sccm}$ Ar flow. The cluster diameter can be varied from 10 to $30 \mathrm{~nm}$ depending on the source parameters, as characterized using scanning electron microscopy (SEM) of deposited clusters. 
(a)

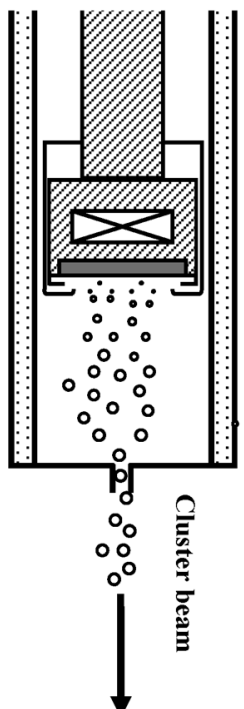

(b)

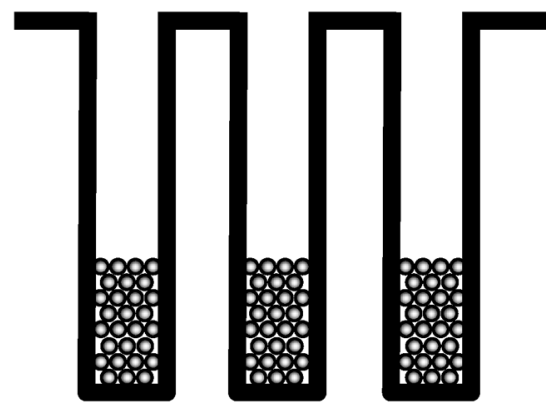

(c)

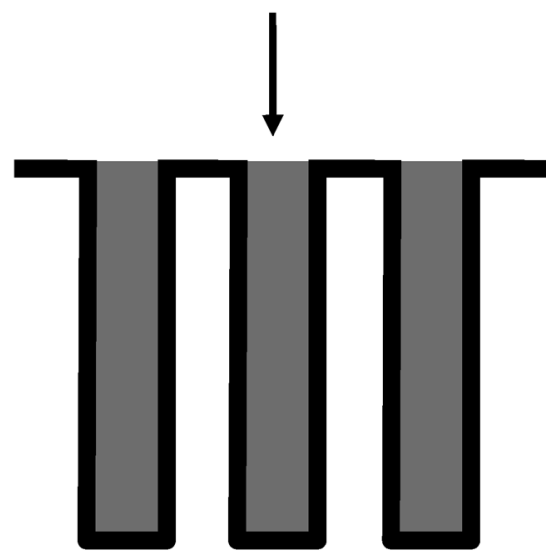

Fig. 1. (a) Clusters are generated in an inert gas aggregation system equipped with magnetron sputtering source. (b) Selective deposition in the trenches is enhanced because clusters can not easily escape from the high aspect ratio structures. (c) After annealing in hydrogen $\left(\sim 5\right.$ torr) at $450{ }^{\circ} \mathrm{C}$ for $2 \mathrm{~h}$, the clusters sinter into the seed layer and form dense metal lines.

The copper clusters were deposited onto various commercially prepared trench structures [Fig. 1(b)], and these samples were subjected to hydrogen annealing to sinter the clusters [Fig. 1(c)]. Three types of trenches and coating were used for this work, namely tantalum nitride (TaN) barriers on $\mathrm{SiO}_{2}$ trenches, ion-induced atomic layer deposition (iALD) TaN barrier/ruthenium $(\mathrm{Ru})$ barrier on low-k dielectric (CORAL) trenches [15], and tantalum (Ta) barrier/copper seed on $\mathrm{SiO}_{2}$ trenches, as summarized in Table I, representing a selection of existing and future barrier/seed technologies. Top-view and cross-sectional SEM images of pre- and postdeposition
TABLE I

Details of the Three DifFerent Dielectric, BARRIER, AND SEEd LaYer COMBINATIONS USED PRIOR TO DISPOSITION

\begin{tabular}{lcl}
\hline \hline Dielectric & $\begin{array}{c}\text { TRENCH DEPTH } \\
(\mathrm{nm})\end{array}$ & Barrier/Seed and the thickness \\
\hline $\mathrm{SiO}_{2}$ & 1000 & $1000 \AA \mathrm{TaN}$ \\
$\mathrm{CORAL} L^{T M}$ & 340 & $25 \AA \mathrm{TaN}$ and $25 \AA \mathrm{Ru}$ \\
$\mathrm{SiO}_{2}$ & 120 & $250 \AA \mathrm{Ta}$ and $1000 \mathrm{~A}$ Cu seed \\
\hline \hline
\end{tabular}

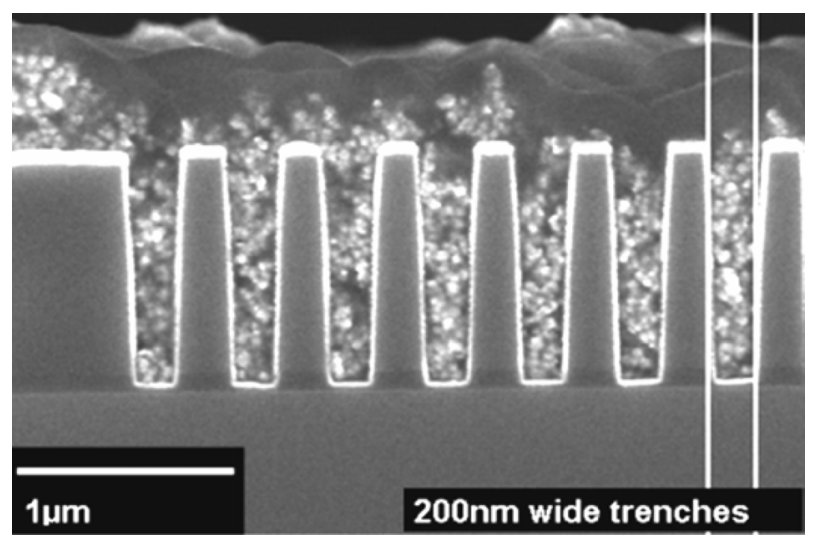

Fig. 2. XSEM image of trenches with a TaN barrier and 5:1 aspect ratio filled with copper clusters. The surface of the sample was covered with photoresist to ensure that the clusters were not disturbed during cleaving and inspection.

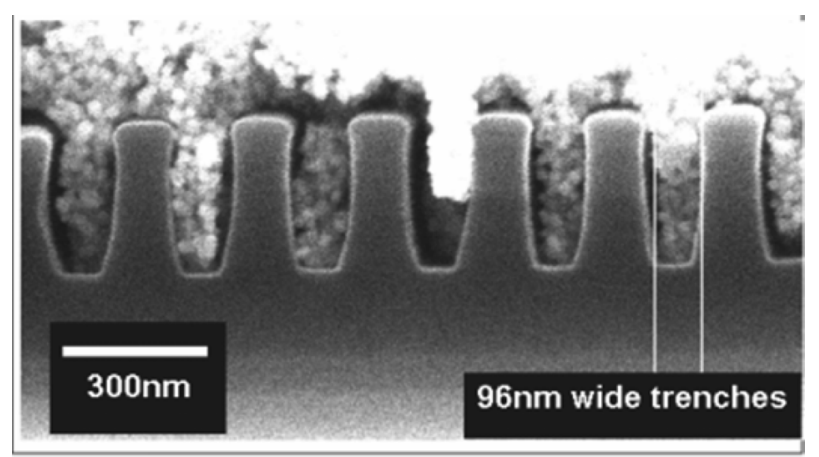

Fig. 3. XSEM of clusters filling $96 \mathrm{~nm}$ wide Ru-coated trenches. The low-k dielectric in the Ru coated sample has deformed during the SEM imaging.

structures have been taken to analyze the results of cluster deposition in these structures.

\section{RESUlTS AND DISCUSSION}

\section{A. Selective Trench Filling}

TaN coated trenches with a high aspect ratio $(5: 1)$ filled with copper clusters are illustrated in Fig. 2. The $200 \mathrm{~nm}$ wide trenches are completely filled with clusters and there is a relatively low density of clusters on the top surface. Similarly, the excellent filling of sub- $100 \mathrm{~nm}$ trenches coated with TaN/Ru barriers is demonstrated in Fig. 3. In this case the trenches have an aspect ratio of $3.5: 1$, but the selectivity is not apparent because the deposition time was longer than necessary, resulting in clusters spilling out of the trenches and spreading across the neighboring plateaus. This assertion is supported by top-view images of large planar regions of the same sample (Fig. 4) which have clean plateaus. To further prove the selectivity, an experiment was carried out with a shorter deposition time. 


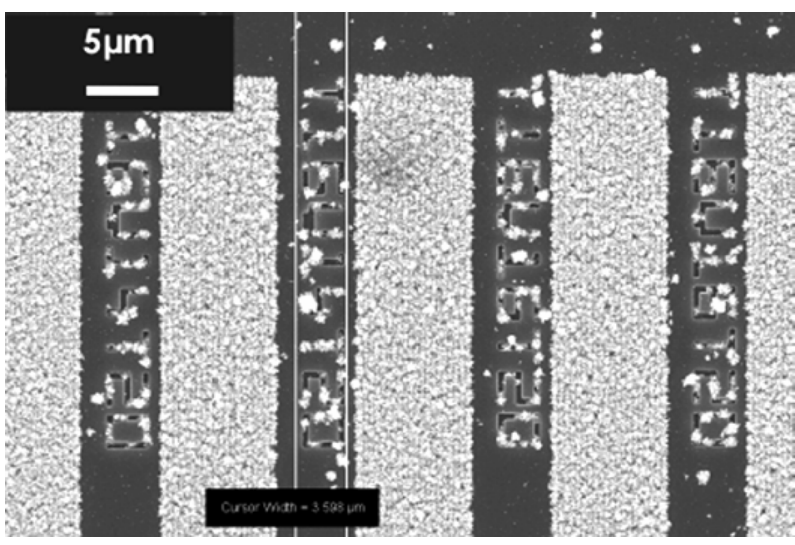

Fig. 4. Large-scale top-view SEM image of the sample shown in Fig. 3, highlighting the selectivity of the deposition.

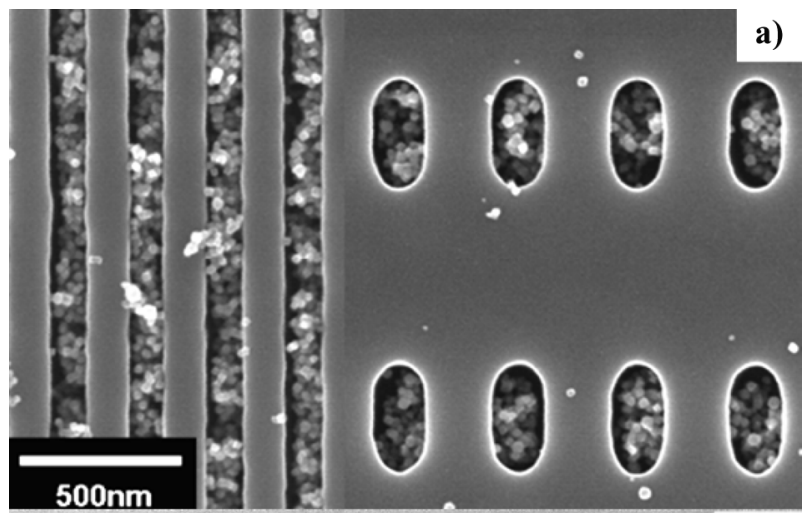

b)

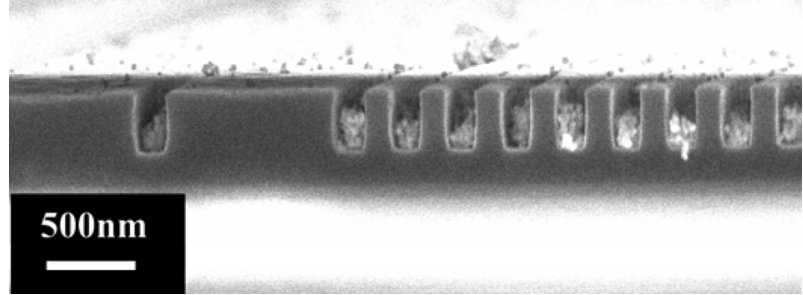

Fig. 5. SEM images of selectively filling sub-200 $\mathrm{nm}$ Ru coated trenches and holes from: (a) top-view and (b) cross-sectional view. Very few clusters adhere to the top planar surface, while the trenches and holes are filled.

Fig. 5 shows that excellent selectivity on a Ru-coated sample, with accumulation in trenches and clean plateaus between them. From the relative cluster coverage on the plateaus and trenches in Fig. 5, a selectivity of at least 500:1 is estimated.

The surfaces of the samples studied were oxidized after being exposed to air and it is clear that the surface state might play an important role in determining the selectivity. However, excellent selectivity (similar to Fig. 4) has also been observed on a Ru sample which has been preannealed in $\mathrm{H}_{2}$ at $200{ }^{\circ} \mathrm{C}$ for $30 \mathrm{~min}$ prior to deposition. After the preannealing, the ruthenium oxide should be reduced to pure ruthenium [16], and yet the selectivity has not been degraded. This proves that the selectivity is not limited to oxidized metal surfaces.

These results indicate that our cluster-based deposition technique has some desirable and unique characteristics. For example, it has the same high purity and directional deposition as ionized physical vapor deposition (iPVD) [17], as well as the selective filling capability of electrochemical deposition (ECD)

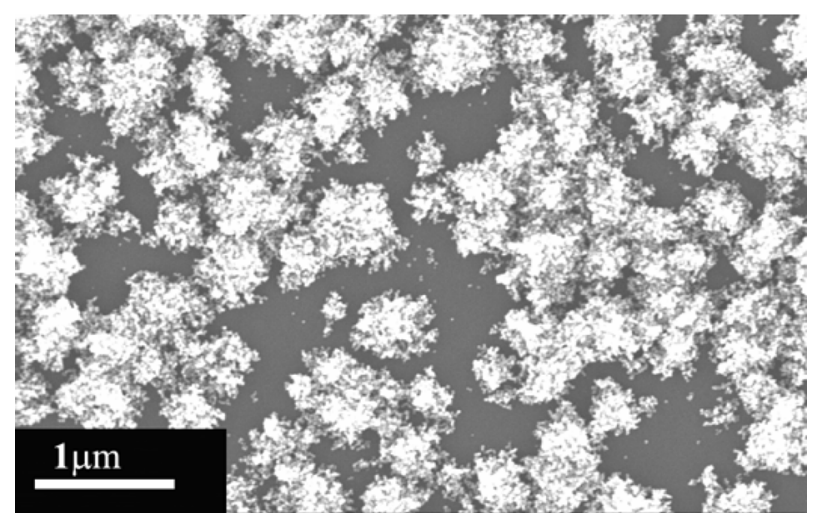

Fig. 6. With long deposition time on planar Ru sample, more clusters have been collected on the plateau. From the distribution of the clusters, this demonstrates that the clusters stick to other clusters better than to the Ru substrate.

[18]. It is generally accepted in the cluster community that clusters generated in similar sources are free from oxidation or other contamination (see, for example, the mass and photoelectron spectra in [4]). Hence, the purity of the $\mathrm{Cu}$ clusters which can in principle be at least as good as in iPVD processes, is in strong contrast with the significant level of impurities that are incorporated into interconnect structures by ECD processes [19]. Although selective filling has been demonstrated in electro-deposition and a chemical vapor deposition (CVD) process [20], this is the first time it has been achieved with a technique which intrinsically produced high purity metal.

\section{B. Mechanism}

We propose the following mechanism for the selective accumulation of clusters at the bottom of the trenches for our deposition technique. Due to the high kinetic energy (at least $2 \mathrm{keV} / \mathrm{cluster}$ ), clusters bounce off the planar surfaces which are perpendicular to the cluster beam but slide along the side walls to the bottom of the trenches. The clusters do not stick where they land because their energy is large enough to overcome the adhesion energy to the surface. The relatively rough or disordered morphology of barrier/seed at the bottom of the trenches may also facilitate the adhesion of clusters. After the arrival of the first clusters at the bottom of the trenches, the accumulation is enhanced because clusters adhere to other clusters efficiently. This effect is shown on a planar surface in Fig. 6. Copper clusters were deposited on a planar Ru sample with double the deposition time compared to the sample in Fig. 5. From the distribution of the clusters, this demonstrates that the clusters stick to other clusters better than to the substrate (cf. antimony clusters in [21]).

The selectivity is better for larger clusters because of their large kinetic energy [5]; however, we have achieved filling of narrow trenches with small (10-15 nm diameter) clusters, showing that the technique is compatible with the requirement of current and future ultralarge-scale integrated (ULSI) technology. More generally the probability of sticking or bouncing is a complex function of the substrate and cluster materials, and the cluster size and velocity [6]. The bouncing behavior observed here was not observed in [7], most likely due to differences in substrate materials and the kinetic energy/atom of the clusters: in the present case the kinetic energies/atom are very small ( $\sim .02 \mathrm{eV} / \mathrm{atom})$, and so the deposition is within what is usually considered the "soft-landing" regime. 

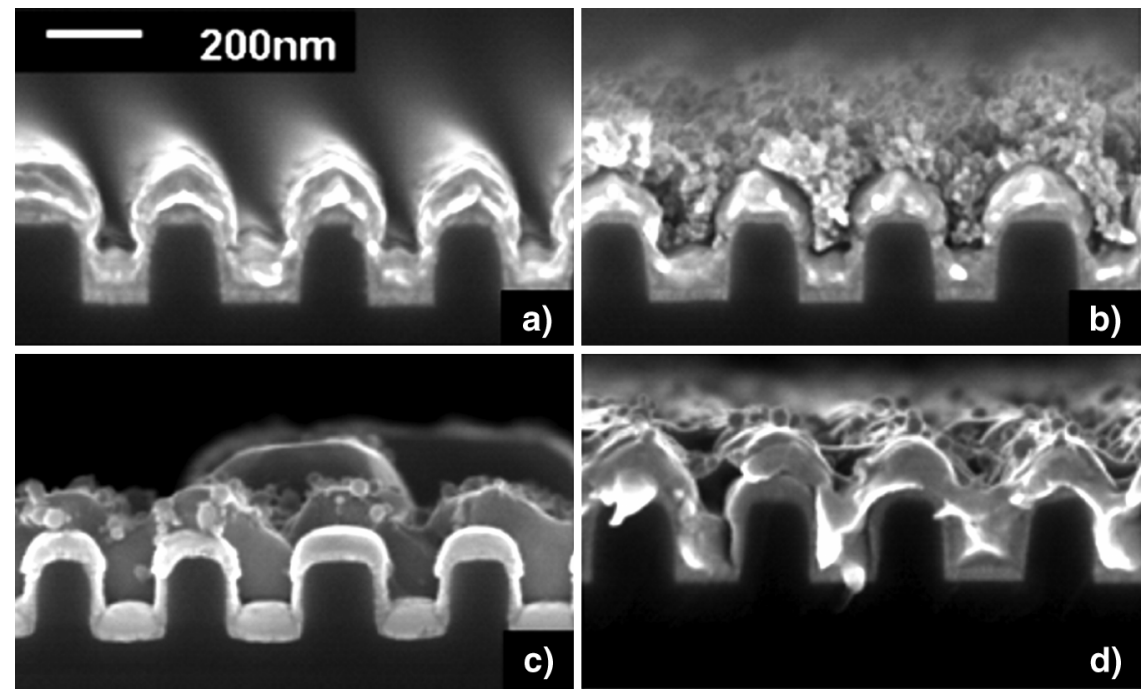

Fig. 7. Results of annealing experiment in $\sim 5$ torr hydrogen at $450{ }^{\circ} \mathrm{C}$ for $2 \mathrm{~h}$. (a) After cleaving but before cluster deposition. (b) After deposition but prior to annealing. (c) After annealing. The cluster material appears to be darker than the barrier/seed layer. (d) After annealing and after additional cleaving. All images showing XSEM of $\mathrm{Ta} / \mathrm{Cu}$ coated trenches are with the same magnification.

\section{Sintering Process}

Clearly the cluster-filled trenches shown in Figs. 2-6 have many voids and would have low electrical conductivity. Therefore, a sintering process [22] is required. We now show that the copper clusters can be sintered into a seed layer by annealing in hydrogen at $450{ }^{\circ} \mathrm{C}$. Initial annealing experiments on Ru seed layers were not successful because the copper clusters failed to adhere to the seed. This was clearly due to oxidation of the $\mathrm{Ru}$ seed, which meant that the $\mathrm{Cu}$ clusters did not wet the seed layer as intended. We therefore turned our attention to trenches with a $\mathrm{Cu}$ seed. Cross-sectional SEM (XSEM) of the cleaved samples before and after deposition are shown in Fig. 7(a) and 7(b) respectively. Since these structures are relatively shallow, it is difficult to optimize the deposition time and the clusters have overflowed from the trenches onto the plateaus (as per discussion of Figs. 3 and 4). The cleaved sample was subjected to annealing at $450{ }^{\circ} \mathrm{C}$ in a $\sim 5$ torr ultrahigh purity hydrogen environment for $2 \mathrm{~h}$. Higher annealing temperatures are expected to cause decomposition of the low-k dielectric. Fig. 7(c) shows that the copper clusters have been effectively sintered after annealing. Since the clusters on the cleaved surface were directly exposed to hydrogen gas during annealing, the wafer was recleaved to demonstrate the effect of sintering within the trenches. Fig. 7(d) shows that, while there is some roughness/damage caused by the cleaving process, the trenches are indeed densely filled with copper.

In a separate experiment, $30 \mathrm{~nm}$ copper clusters were deposited on a planar sample with copper seed. After ( $\sim 5$ torr) hydrogen annealing at $450{ }^{\circ} \mathrm{C}$ for $2 \mathrm{~h}$, the sheet resistance was obtained using four-terminal resistance measurement. Preliminary results indicate that the resistivity of $100 \mathrm{~nm}$ thick annealed cluster film is around $2.3 \times 10^{-8} \Omega \mathrm{m}$. This is reasonably close to the bulk resistivity $\left(1.6 \times 10^{-8} \Omega \mathrm{m}\right)$ and the value required by industry $\left(2.2 \times 10^{-8} \Omega \mathrm{m}\right)$ [1]. The details of the resistivity measurement will be reported elsewhere.

\section{CONCLUSION}

In summary, we have demonstrated that reflection of clusters can be employed to achieve selective filling of high-aspect-ratio
(5:1) damascene trenches with copper. In that case, the CMP issues such as dishing or erosion would be minimized, thus leading to better process integration. Annealing in hydrogen greatly improves the quality of the deposited material. Preliminary results show that the resistivity is around $2.3 \times 10^{-8} \Omega \mathrm{m}$. This technique has great potential to meet the requirements for ULSI interconnect metallization. Future work will focus on direct measurements of the electrical conductivity of the annealed copper material within the trenches, and reliability testing (stress-migration and electromigration).

\section{ACKNOWLEDGMENT}

The authors would like to acknowledge useful discussion with, and provision of wafers by, Novellus, Texas Instruments and Sematech.

\section{REFERENCES}

[1] International Technology Roadmap for Semiconductors (Update) 2006 [Online]. Available: http://public.itrs.net

[2] M. Fayolle, G. Passemard, O. Louveau, F. Fusalba, and J. Cluzel, "Challenges of back end of the line for sub $65 \mathrm{~nm}$ generation," Microelectron. Eng., vol. 70, pp. 255-266, 2003.

[3] S. Wolf, Silicon Processing for the VLSI Era: Deep-Submicron Process Technology. Sunset Beach, CA: Lattice Press, 2002, vol. 4.

[4] W. A. de Heer, "The physics of simple metal clusters: Experimental aspects and simple models," Rev. Mod. Phys., vol. 65, p. 611, 1993.

[5] J. G. Partridge, S. A. Brown, A. D. F. Dunbar, R. Reichel, M. Kaufmann, C. Siegert, S. Scott, and R. J. Blaikie, "Templated-assembly of conducting antimony cluster wires," Nanotechnology, vol. 15, pp. 1382-1387, 2004.

[6] A. Awasthi, S. C. Hendy, P. Zoontjens, and S. A. Brown, "Reentrant adhesion behavior in nanocluster deposition," Phys. Rev. Lett., vol. 97, p. 186103, 2006.

[7] H. Haberland, M. Mall, M. Moseler, Y. Qiang, T. Reiners, and Y. Thurner, "Filling of micrometer-sized contact holes with copper by energetic cluster impact," J. Vac. Sci. Technol. A: Vac., Surf., Films, vol. 12 , pp. $2925-2930,1994$.

[8] P. Gatz and O. F. Hagena, "Cluster beams for metallization of microstructured surfaces," Appl. Surf. Sci., vol. 91, pp. 169-174, 1995.

[9] T. Miyake, H. Petek, K. Takeda, and K. Hinode, "Atomic hydrogen enhanced reflow of copper," Appl. Phys. Lett., vol. 70, pp. 1239-1241, 1997.

[10] K. K. Choi, J. H. Yun, and S. W. Rhee, "Effect of hydrogen remote plasma annealing on the characteristics of copper film," Thin Solid Films, vol. 429, pp. 255-260, 2003.

[11] O. H. Nielsen, J. P. Sethna, P. Stoltze, K. W. Jacobsen, and J. K. Norskov, "Melting a copper cluster-Critical-droplet theory," Europhys. Lett., vol. 26, pp. 51-56, 1994. 
[12] L. Wang, Y. Zhang, X. Bian, and Y. Chen, "Melting of Cu nanoclusters by molecular dynamics simulation," Phys. Lett. A, vol. 310, pp. 197-202, 2003.

[13] R. Reichel, J. G. Partridge, A. D. F. Dunbar, S. A. Brown, O. Caughley, and A. Ayesh, "Construction and application of a UHV compatible cluster deposition system," J. Nanoparticle Res., vol. 8, pp. 405-416, 2006.

[14] H. Haberland, M. Karrais, M. Mall, and Y. Thurner, "Thin-films from energetic cluster impact-A feasibility study," J. Vac. Sci. Technol. A Vac. Surf., Films, vol. 10, pp. 3266-3271, 1992.

[15] X. P. Qu, J. J. Tan, M. Zhou, T. Chen, Q. Xie, G. P. Ru, and B. Z. Li, "Improved barrier properties of ultrathin Ru film with TaN interlayer for copper metallization," Appl. Phys. Lett., vol. 88, 2006.

[16] M. Hiratani, Y. Matsui, K. Imagawa, and S. Kimura, "Hydrogen reduction properties of RuO2 electrodes," Jpn. J. Appl. Phys. Pt. 2-Lett., vol. 38, pp. L1275-L1277, 1999.

[17] C. A. Nichols, S. M. Rossnagel, and S. Hamaguchi, "Ionized physical vapor deposition of $\mathrm{Cu}$ for high aspect ratio damascene trench fill applications," J. Vac. Sci. Technol. B, vol. 14, p. 3270, 1996.

[18] D. Josell, D. Wheeler, C. Witt, and T. P. Moffat, "Seedless superfill: Copper electrodeposition in trenches with ruthenium barriers," Electrochem. Solid State Lett., vol. 6, pp. C143-C145, 2003.

[19] M. Stangl, V. Dittel, J. Acker, V. Hoffmann, W. Gruner, S. Strehle, and $\mathrm{K}$. Wetzig, "Investigation of organic impurities adsorbed on and incorporated into electroplated copper layers," Appl. Surf. Sci., vol. 252, pp. 158-161, 2005.

[20] P. Hyungsang, K. Wonyong, C. Seung-Man, P. Ki-Chul, K. Ho-Kyu, M. Joo-Tae, S. Kyuchan, L. Hyunbae, K. Ohgyum, and K. Sangwon, "Superfilling CVD of copper using a catalytic surfactant," presented at the Int. Interconnect Technology Conf., Burlingame, CA, 2001, unpublished.

[21] A. D. F. Dunbar, J. G. Partridge, M. Schulze, and S. A. Brown, "Morphological differences between $\mathrm{Bi}, \mathrm{Ag}$ and $\mathrm{Sb}$ nano-particles and how they affect the percolation of current through nano-particle networks," Eur. Phys. J. D, vol. 39, pp. 415-422, 2006.

[22] M. Yeadon, J. C. Yang, R. S. Averback, J. W. Bullard, D. L. Olynick, and J. M. Gibson, "In-situ observations of classical grain growth mechanisms during sintering of copper nanoparticles on (001) copper," Appl. Phys. Lett., vol. 71, pp. 1631-1633, 1997.

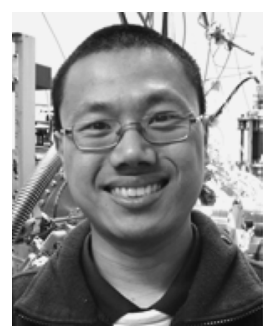

Kheng Chok Tee received the B.Eng. (Hons.) and M. Eng. degrees in electrical and electronics engineering from Nanyang Technological University, Singapore, in 1997 and 1999, respectively. Currently he is working toward the Ph.D. degree at the University of Canterbury, Christchurch, New Zealand.

He has since worked as a Technology Development Engineer in Chartered Semiconductor Manufacturing Ltd. for five years. His research interests include transistor development and copper interconnect. He holds more than 15 U.S. patents.

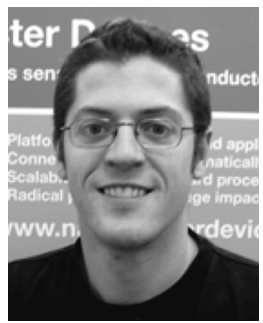

Andreas Lassesson received the M.Sc. and Ph.D degrees in physics from Gothenburg University, Sweden in 1998 and 2003, respectively.

He has since then conducted postdoctoral research on cluster physics at the University of Greifswald, Germany, and is now working as a Research Scientist with the development of nanodevices, self-assembled from atomic clusters, for Nano Cluster Devices Ltd., Christchurch, New Zealand.

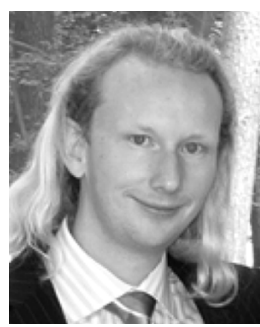

Joris van Lith received the M.Sc. and Ph.D. degrees in applied physics from the University of Twente, The Netherlands, in 2000 and 2005, respectively.

His research focused on integrated optics for both telecom and sensing applications. Currently he is working on devices based on nanofabrication and cluster assembly methods at Nano Cluster Devices Ltd., Christchurch, New Zealand.

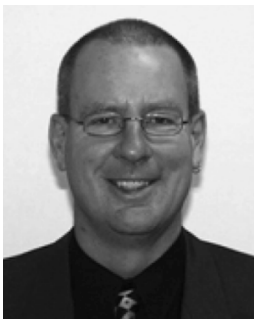

Simon A. Brown received the B.Sc. (Hons.) degree from Victoria University, Wellington, New Zealand, in 1986 and the Ph.D. degree from the University of Cambridge, U.K., in 1990.

Since 1998 he has been on the faculty of the Department of Physics and Astronomy at the University of Canterbury (UC), Christchurch, New Zealand. He is a Founding Member of Nanostructure Engineering Science and Technology (NEST) Group at UC and he has recently been heavily involved in the formation of a national Centre of Research Excellence, the MacDiarmid Institute for Advanced Materials and Nanotechnology. He has published in fields as diverse as magnetism, resonant tunneling, the quantum Hall effect, and the optical properties of Antarctic sea ice. He now leads a group whose focus is the fabrication of cluster assembled nanoelectronic devices.

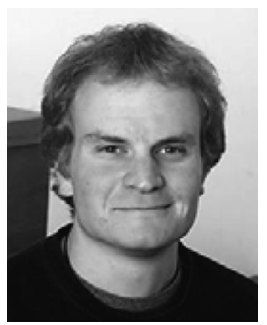

Jim G. Partridge received the B.Sc. (Hons.) degree in physics and the Ph.D. degree from the University of Bath, U.K., in 1996 and 2000, respectively.

He has since conducted postdoctoral research on submillimeter-wave device fabrication at the University of Bath and developed nanofabrication/cluster assembly methods with the Nanostructure Engineering Science and Technology (NEST) Group, University of Canterbury, Christchurch, New Zealand. He is currently investigating novel high-k RMIT University, Melbourne. dielectric layers and thin-film metal-oxide devices at

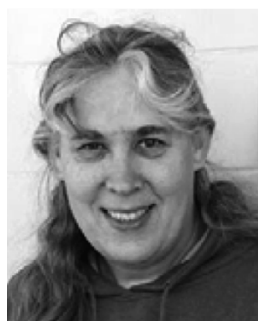

Monica Schulze received the B.Sc. degree in physics in 2000 from the University of Canterbury, Christchurch, New Zealand.

Currently she is working on devices based on nanofabrication at Nano Cluster Devices Ltd., Christchurch, New Zealand

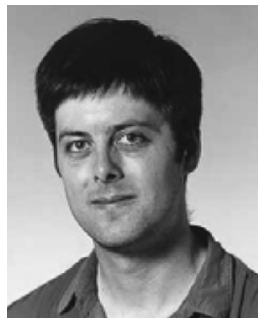

Richard J. Blaikie (M'93) received the B.Sc. (Hons.) degree from the University of Otago, New Zealand, in 1988 and the Ph.D. degree in physics from the University of Cambridge, U.K., in 1992.

For one year, he was a visiting scientist at the Hitachi Cambridge Laboratory, investigating single-electron transport effects in semiconductor nanostructures. He returned to New Zealand in 1993, taking up a position in the Department of Electrical and Electronic Engineering at the University of Canterbury, and he is currently a Professor. He is a founding member of the University's Nanostructure Engineering, Science and Technology (NEST) group, and is currently the Deputy Director of the MacDiarmid Institute for Advanced Materials and Nanotechnology, a New Zealand Centre of Research Excellence (formed in collaboration between a number of Universities and Crown Research Institutes). His principal research interests are the development of low-cost nanolithography techniques using near field illumination, and the utilization of subwavelength structures at sub-mm and visible wavelengths. This applied electromagnetics research led to the award of the 2002 T. K. Sidey Medal of the Royal Society in New Zealand. His research interests also include polarization modulation in optical communications systems, modeling of semiconductor device structures, and the application of nanofabrication techniques to new electronic, optical, chemical, and biological devices. 